\title{
Synthesis and antibacterial activity of tripropeptin C derivatives modified at the carboxyl groups
}

\author{
Sehei Hirosawa ${ }^{1}$, Yoshiaki Takahashi ${ }^{1}$, Hideki Hashizume ${ }^{2}$, Toshiaki Miyake ${ }^{1}$ and Yuzuru Akamatsu ${ }^{2}$
}

The Journal of Antibiotics (2014) 67, 265-268; doi:10.1038/ja.2013.128; published online 4 December 2013

Keywords: antibacterial activity; MRSA; synthesis; tripropeptin

In recent years, there has been a worrying increase in the number of infections caused by drug-resistant bacteria. Therapeutic agents traditionally used to treat these bacterial infections are becoming increasingly ineffective. ${ }^{1,2}$ There is therefore an urgent need for the discovery and development of new antibacterial agents that are effective against multidrug-resistant bacteria, as well as being structurally different from the existing agents and capable of exerting their inhibitory activity according to a novel mode of action. ${ }^{3}$

We have screened for novel antibiotics that are active against drugresistant bacteria from microbial origins. As part of this program, we have discovered a structurally analogous mixture of tripropeptins, ${ }^{4-6}$ which are a group of novel cyclic lipodepsipeptide antibiotics, in the cultured cells and broth of the soil bacterium Lysobacter sp. strain BMK333-48F3. Tripropeptins consist of a cyclic octapeptide core and a fatty acyl side chain. Based on differences in their fatty acyl side chains, tripropeptins can be classified into six different components (that is, A-E and Z).

Its major component, tripropeptin C (TPPC), showed potent antimicrobial activities toward a variety of different Gram-positive pathogens, including methicillin-resistant Staphylococcus aureus (MRSA), vancomycin-resistant Enterococcus faecalis/faecium (VRE) and penicillin-resistant Streptococcus pneumoniae. ${ }^{4,5,7}$ Plusbacins ${ }^{8,9}$ and empedopeptin, ${ }^{10,11}$ which are cyclic lipodepsipeptide antibiotics structurally related to TPPC, have also been reported to exhibit potent activities toward Gram-positive bacteria. TPPC also showed excellent in vivo therapeutic efficacy in a mouse-MRSA septicemia model when administered intravenously and its $\mathrm{ED}_{50}$ value was comparable to that of vancomycin. Furthermore, TPPC exhibited a favorable toxicological profile that included no acute toxicity and no 14-day repeated toxicities in mice when administered intravenously. ${ }^{7}$

TPPC inhibited peptidoglycan biosynthesis in a different way than the drugs that currently target peptidoglycan biosynthesis, including vancomycin and bacitracin, and showed no cross-resistance to these drugs. ${ }^{12}$ Based on its excellent biological properties, TPPC is considered to be a promising novel class of antibiotic against MRSA and vancomycin-resistant Enterococcus faecalis/faecium.
In TPPC, there are five hydroxyl groups, two carboxyl groups and a guanidine group. To investigate the detailed structure-activity relationships of TPPC, we have focused our efforts on exploring these functional groups. As outlined in Scheme 1, this paper describes the role of carboxylic groups for the antibacterial activity. In this initial efforts, the modification of the two carboxyl groups of TPPC in the form of esters and amides and their antibacterial activities are described.

The esterification reaction was performed under mild reaction conditions, where TPPC was treated with an excess of (trimethylsilyl)diazomethane $\left(\mathrm{TMSCHN}_{2}\right)$ in $\mathrm{MeOH}$ to give the two separable mono-methyl esters 1 and $2\left(\mathrm{OCH}_{3}\right.$ signal at $\delta 3.68$ for $\mathbf{1}$ and at $\delta 3.53$ for 2, refer to Supplementary Information) in $15 \%$ and $28 \%$ yields, respectively, together with two unidentified products. In contrast, the reaction of TPPC with diphenyldiazomethane $\left(\mathrm{Ph}_{2} \mathrm{CN}_{2}\right.$, 1.5 equiv. relative to the TPPC) afforded the two mono-diphenylmethyl esters $3(25 \%)$ and $4(18 \%)$, together with a $50 \%$ recovery of TPPC. The extension of the reaction time, as well as an increase in the charge of $\mathrm{Ph}_{2} \mathrm{CN}_{2}$ did not lead to any improvement in the yield of the corresponding esters. Interestingly, the reaction of the $p$-toluenesulfonate salt ${ }^{13}$ of TPPC with a large excess of TMSCHN $\mathrm{T}_{2}$ or $\mathrm{Ph}_{2} \mathrm{CN}_{2}$ gave the bis-methyl ester $\mathbf{5}$ or bis-diphenylmethyl ester $\mathbf{6}$ as the major product, respectively. Based on these results, it was suggested that TPPC behaved in a similar way to amino acids, in which it existed in the zwitterionic form under these reaction conditions.

It was difficult to determine the structures of compounds 1 and 2 by NMR analysis with HMBC and NOESY techniques. Therefore, we attempted to confirm their structures by amino-acid analysis using Marfey's method. ${ }^{14}$ Using this method, it should be possible to detect threo- $\beta$-hydroxy-D-aspartic acid from the methyl ester at the C-4 position, but not from the methyl ester at the C-16 position. Thus, compounds 1 and 2 were converted to the corresponding alcohols $\mathbf{1 2}$ and 13, respectively, following the reduction of their ester and lactone moieties with $\mathrm{NaBH}_{4}$ as shown in Scheme 2.

Compounds 12, 13 and TPPC were then hydrolyzed in refluxing 6 $\mathrm{M}$ aqueous $\mathrm{HCl}$ for $20 \mathrm{~h}$ to give the corresponding constituent amino 


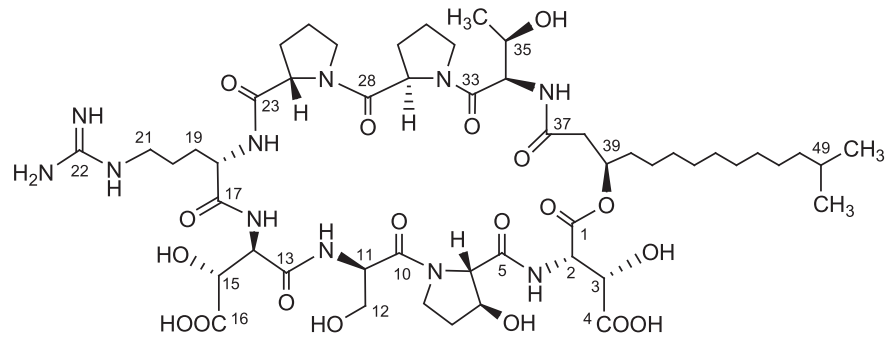

Tripropeptin C (TPPC)

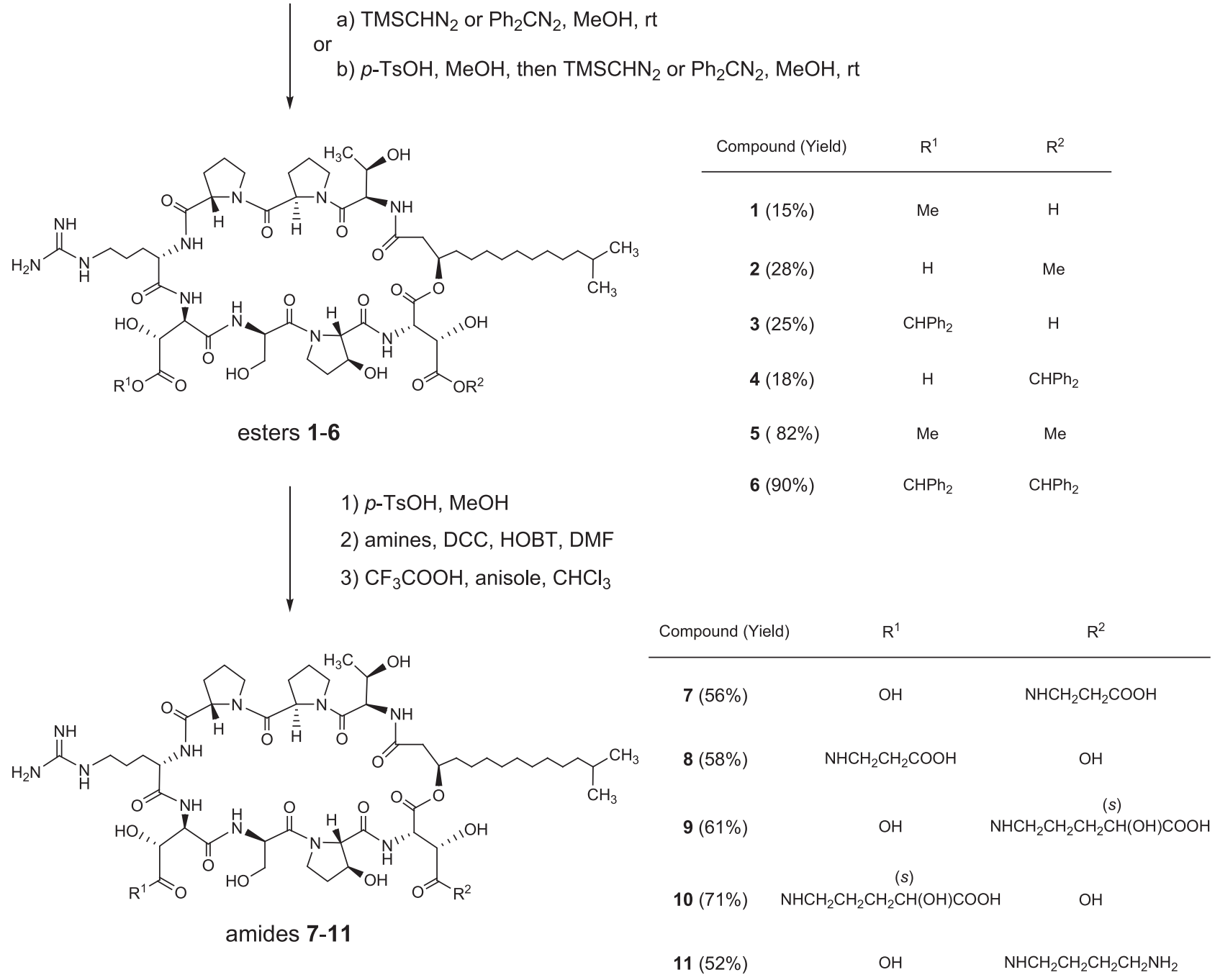

Scheme 1 The synthetic procedures for preparation of TPPC derivatives modified at the carboxyl groups.

acids. The acidic hydrolysates were treated with 1-fluoro-2,4-dinitrophenyl-5-L-alanine amide to give the corresponding Marfey's derivatives, which were analyzed by HPLC. The amino-acid derivatives were identified through a comparison of their retention times with those of the Marfey's derivatives of the authentic amino acids derived from TPPC and standard threo- $\beta$-hydroxy-L-aspartic acid. As expected, the Marfey's derivative of threo- $\beta$-hydroxy-D-aspartic acid was detected from 13 . In contrast, the threo- $\beta$-hydroxy-L-aspartic acid and threo- $\beta$ hydroxy-D-aspartic acid Marfey's derivatives were not detected from
12. These results therefore revealed that 1 and 2 were the $16-$ and 4-methyl esters of TPPC, respectively.

The structures of $\mathbf{3}$ and $\mathbf{4}$ were determined by converting these compounds to the corresponding mono-methyl esters. Treatment of the p-toluenesulfonate salts of $\mathbf{3}$ and $\mathbf{4}$ with (trimethylsilyl)diazomethane in $\mathrm{MeOH}$, followed by the removal of the diphenylmethyl ester portion using trifluoroacetic acid, gave compounds 2 and 1, respectively.

Preparation of amides was achieved by using the $p$-toluenesulfonate salts of the mono-diphenylmethyl ester 3 or 4 . The condensation 


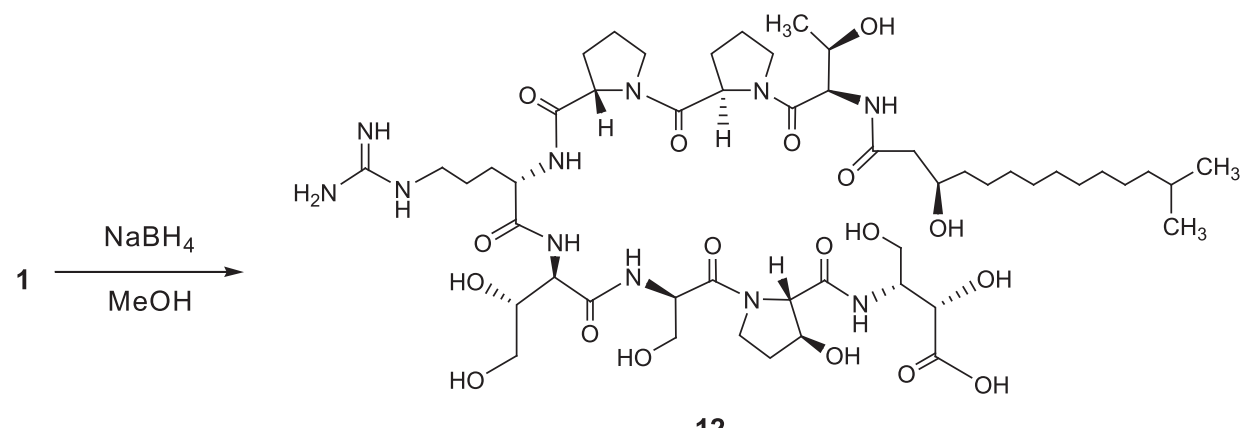

12

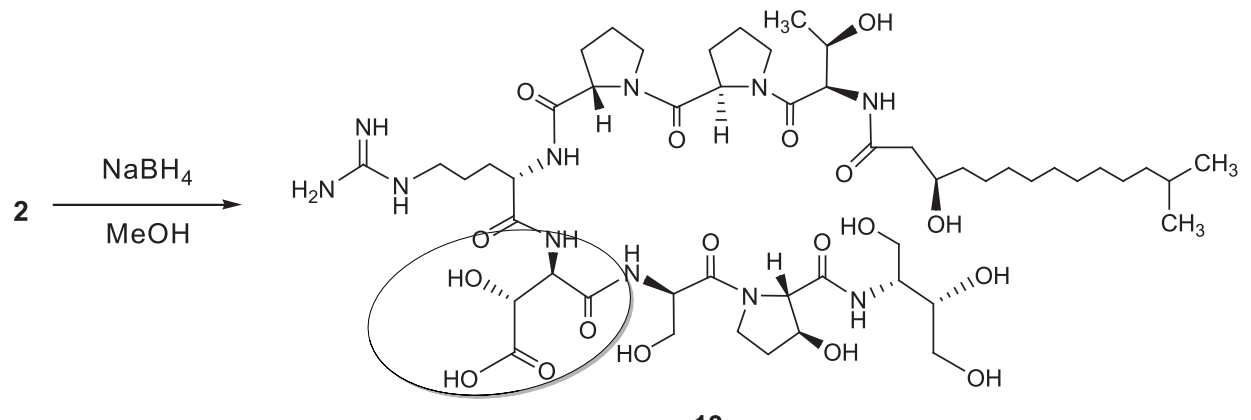

13

Scheme 2 Reductive products (12 and 13) for amino-acid analysis. (Threo- $\beta$-hydroxy-D-aspartic acid portion in 13 is highlighted.)

reactions were effectively conducted with the appropriate amines using 1-hydroxybenzotriazole and $N, N^{\prime}$-dicyclohexylcarbodiimide. Subsequent removal of the protecting group with trifluoroacetic acid afforded amides $7-11$ as the $p$-toluenesulfonate salts in $52-71 \%$ yields from 3 or 4 .

Table 1 presents the antibacterial activities of the ester and amide derivatives of TPPC against the clinical isolates of methicillin-sensitive S. aureus (MSSA, $n=10$ ) and methicillin-resistant S. aureus (MRSA, $n=10)$.

The antibacterial activities of the mono-esters $1-4$ were less than that of TPPC. Moreover, the bis-esters $\mathbf{5}$ and $\mathbf{6}$ suffered substantial reductions in their antibacterial activity. TPPC is known to bind to undecaprenyl pyrophosphate via a calcium ion, with the resulting complex inhibiting peptidoglycan biosynthesis. ${ }^{12}$ In contrast, however, it was not possible for the bis-methyl ester $\mathbf{5}$ to form a biologically important complex such as the one formed in the case of TPPC, as shown in Supplementary Figure S1. Based on these results, it became clear that the two free carboxyl groups of TPPC were critical for the binding of TPPC to the target undecaprenyl pyrophosphate molecule, and important for the expression and strength of its antibacterial activity.

In contrast to esters $\mathbf{1 - 6}$, amides $\mathbf{7 - 1 0}$, which were synthesized via the introduction of different amino-acid groups at the C-4 or C-16 position, contained two free carboxyl groups. We were interested in these compounds because they possessed the two free carboxyl groups, which may be meaningful and it was expected that these groups would impart high levels of antibacterial activity. Unfortunately, however, the activities of these compounds greatly reduced compared with TPPC. Furthermore, the antibacterial activity of amide 11, which had an amino side chain and only one free carboxylic acid group, was much lower than the activities of the corresponding amides 7 and $\mathbf{9}$, which had the two carboxylic acid groups.
Table 1 Antibacterial activities of ester and amide derivatives of TPPC

\begin{tabular}{|c|c|c|c|c|c|c|}
\hline \multirow[b]{3}{*}{ Compound } & \multicolumn{6}{|c|}{$M I C\left(\mu g m I^{-1}\right)$} \\
\hline & \multicolumn{3}{|c|}{$\begin{array}{l}\text { Methicillin-sensitive Staphylococ- } \\
\text { cus aureus (MSSA) } \\
\text { (clinical isolates, } 10 \text { strains) }\end{array}$} & \multicolumn{3}{|c|}{$\begin{array}{c}\text { Methicillin-resistant Staphylococ- } \\
\text { cus aureus (MRSA) } \\
\text { (clinical isolates, } 10 \text { strains) }\end{array}$} \\
\hline & Range & $M I C_{50}$ & $M C_{90}$ & Range & $M / C_{50}$ & $M I C_{90}$ \\
\hline 1 & $3.13-6.25$ & 3.13 & 6.25 & $6.25-12.5$ & 6.25 & 12.5 \\
\hline 2 & $6.25-12.5$ & 6.25 & 12.5 & 12.5 & 12.5 & 12.5 \\
\hline 3 & $1.56-3.13$ & 3.13 & 3.13 & $3.13-6.25$ & 3.13 & 6.25 \\
\hline 4 & $12.5-50$ & 12.5 & 50 & 25-50 & 25 & 50 \\
\hline 5 & 100 & 100 & 100 & 100 & 100 & 100 \\
\hline 6 & $25-100$ & 100 & 100 & $50-100$ & 100 & 100 \\
\hline 7 & $12.5-50$ & 25 & 25 & $6.25-50$ & 25 & 50 \\
\hline 8 & 50 & 50 & 50 & 25-50 & 50 & 50 \\
\hline 9 & $3.13-12.5$ & 12.5 & 12.5 & $1.56-12.5$ & 6.25 & 12.5 \\
\hline 10 & $12.5-50$ & 50 & 50 & $6.25-50$ & 50 & 50 \\
\hline 11 & 50 & 50 & 50 & $25-50$ & 50 & 50 \\
\hline TPPC & $0.78-1.56$ & 0.78 & 0.78 & $0.39-1.56$ & 0.78 & 1.56 \\
\hline
\end{tabular}

Abbreviation: TPPC, tripropeptin C.

In conclusion, we have made a series of modifications to the carboxyl groups of TPPC, and synthesized the corresponding esters and amides as a part of the structure-activity relationship study. The structures of mono-esters obtained were confirmed by amino-acid analysis using Marfey's method. Unfortunately, all of the modifications reported in the current paper led to a reduction in the antibacterial activity compared with TPPC and therefore highlighted the importance of the two free carboxyl groups to the activity of 
TPPC. As a follow-up to this program, work toward modifying the terminal guanidine group of the arginine residue in TPPC is currently underway in our laboratory.

\section{ACKNOWLEDGEMENTS}

We greatly appreciate the assistance of Dr Yoshihiko Kobayashi of the Institute of Microbial Chemistry (BIKAKEN), Hiyoshi, for constructive discussions. We are also grateful to Kunio Inoue of the Institute of Microbial Chemistry (BIKAKEN), Tokyo, for determining the spectra of the antibacterial compounds and Dr Ryuichi Sawa of the Institute of Microbial Chemistry (BIKAKEN), Tokyo, for the MS experiments.

1 Rice, L. B. The clinical consequences of antimicrobial resistance. Curr. Opin. Microbiol. 12, 476-481 (2009).

2 Cai, J. C. et al. Linezolid-resistant clinical isolates of meticillin-resistant coagulasenegative staphylococci and Enterococcus faecium from China. J. Med. Microbiol. 61 1568-1573 (2012)

3 Sawa, R. et al. Amycolamicin: a novel broad-spectrum antibiotic inhibiting bacterial topoisomerase. Chem. Eur. J. 18, 15772-15781 (2012).
4 Hashizume, H. et al. Tripropeptins, novel antimicrobial agents produced by Lysobacter sp. I. Taxonomy, isolation and biological activities. J. Antibiot. 54, 1054-1059 (2001).

5 Hashizume, H. et al. Tripropeptins, novel antimicrobial agents produced by Lysobacter sp. II. Structure elucidation. J. Antibiot. 57, 52-58 (2004).

6 Hashizume, H., Hattori, S., Igarashi, M. \& Akamatsu, Y. Tripropeptin E, a new tripropeptin group antibiotic produced by Lysobacter sp. BMK333-48F3. J. Antibiot. 57, 394-399 (2004).

7 Hashizume, H. et al. Tripropeptin C blocks lipid cycle of peptidoglycan synthesis by complex formation with undecaprenyl pyrophosphate. 50th Interscience Conference on Antimicrobial Agents and Chemotherapy, Boston, Poster F-1611 (2010).

8 Shoji, J. et al. Isolation and characterization of new peptide antibiotics, plusbacins A1A4 and B1-B4. J. Antibiot. 45, 817-823 (1992).

9 Shoji, J. et al. Structure of new peptide antibiotics, plusbacins A1-A4 and B1-B4. J. Antibiot. 45, 824-831 (1992).

10 Konishi, M. et al. Empedopeptin (BMY-28117), a new depsipeptide antibiotic. I. Production, isolation and properties. J. Antibiot. 37, 949-957 (1984).

11 Sugawara, K., Numata, K., Konishi, M. \& Kawaguchi, H. Empedopeptin (BMY-28117) a new depsipeptide antibiotic. II. Structure determination. J. Antibiot. 37, 958-964 (1984).

12 Hashizume, H. et al. Tripropeptin C blocks the lipid cycle of cell wall biosynthesis by complex formation with undecaprenyl pyrophosphate. Antimicrob. Agents Chemother. 55, 3821-3828 (2011).

13 Aboderin, A. A., Delpierre, G. R. \& Fruton, J. S. Benzhydryl esters of amino acids in peptide synthesis. J. Am. Chem. Soc. 87, 5469-5472 (1965).

14 Murakami, M., Itou, Y., Isida, K. \& Shin, H. J. Prenylagaramides A and B, new cyclic peptides from two strains of Oscillatoria agardhii. J. Natl Prod. 62, 752-755 (1999).

Supplementary Information accompanies the paper on The Journal of Antibiotics website (http://www.nature.com/ja) 